\title{
How does working memory store more information at larger set
}

\section{sizes? A composite code model.}

\author{
Rob Udale ${ }^{1}$, Katerina Gramm², Masud Husain ${ }^{23}$, Sanjay G. Manohar 23 \\ 1 Department of Psychology, University of Sheffield, United Kingdom. \\ 2 Department of Experimental Psychology, University of Oxford, United Kingdom. \\ 3 Nuffield Department of Clinical Neurosciences, University of Oxford, United Kingdom.
}

Word count: 9,729

\begin{abstract}
Author Note.
The research was funded by a Wellcome Trust Principal Research Fellowship to MH and the NIHR MRC Clinician scientist fellowship MR/P00878/X to SGM

Oxford Biomedical Research Centre.

We have no known conflict of interest to disclose.

Correspondence concerning this article should be addressed to Rob Udale, Department of Psychology, University of Sheffield.

Contact: $\underline{\text { r.c.udale@sheffield.ac.uk }}$
\end{abstract}


The conjunctive code model

\section{Abstract}

A central feature of working memory is its limited capacity in terms of the amount of information that can be simultaneously maintained. Despite this, many studies observe an increase in the total amount when more items are maintained (set size), as measured by Shannon information. We propose the composite code model which maintains this fixed capacity assumption but demonstrates increasing observed information across set sizes. This relies on the hierarchical organisation of the visual system, in which higher-order information is abstracted about simple study displays. Using Bayesian inference, target responses can be inferred from knowledge about nontargets. We tested this model against our own data from a delayed reproduction task and those of published open data sets. We found initial support for the model, with its predictions matching those of the observed effects.

Keywords: "Working memory", "short-term memory", "visual system", "composite code", "delayed reproduction", "delayed estimation", "computational model", "representation", "memory load", "visual hierarchy", "compression", "information", "set size".

\section{Introduction}

A widely accepted central property of working memory (WM), the store that allows temporary maintenance and manipulation of information held in mind, is that it is severely limited in capacity (Atkinson \& Shiffrin, 1968; Broadbent, 1958; Baddeley \& Hitch, 1974; Cowan, 2001; Jacobs, 1887; Miller, 1956; Pashler, 1988). This idea is well supported by many well-established effects, such as the low performance for large memory arrays (Sperling, 1960), rapid forgetting when rehearsal mechanisms are interrupted (Peterson \& Peterson, 1959), and near-perfect recall for items at the end of a list (Phillips, Shiffrin \& Atkinson, 1967). Having a fixed capacity is a core component of the 
The conjunctive code model

vast majority of WM models (Atkinson \& Shiffrin, 1968; Baddeley \& Hitch, 1974; Baddeley, 2000; Broadbent, 1958; Cowan, 2001; Luck \& Vogel, 1997; Oberauer, 2002; Pylyshyn, 1989). A central tenet to many of these models is that this limited capacity is a fixed parameter that remains constant within individuals across different experimental conditions, time, and settings. In this paper, we highlight that many modern studies do not in fact observe a fixed WM capacity: total information capacity appears to reliably increase with memory load (Bays \& Husain, 2008; van den Berg et al., 2012), and we propose a potential biologically plausible explanation.

The set size effect, which is the decline in memory performance as the number of items to be remembered increases, has been one of the key benchmarks for constraining models of WM (Cowan, 2001; Luck \& Vogel, 1997; Oberauer et al., 2018; van den Berg et al., 2015). A widely used task to measure the set size effect is the delayed reproduction task (Wilken \& Ma, 2004), in which participants are asked to reproduce the continuous feature (e.g., colour, location, or orientation) of one of the studied objects, probed by a cue (Bays, Catalao \& Husain, 2009; Wilken \& Ma, 2004; Zhang \& Luck, 2008). Performance in this task shows a continuous decrement in performance with each additional item encoded, leading theorists to propose that WM maintenance relies on a pool of divisible resources (Bays \& Husain, 2008; Wilken \& Ma, 2004) in contrast to slot-based models which predicted near-ceiling performance up to about 3-4 maintained items (Cowan, 2001; Luck \& Vogel, 1997; Pashler, 1988). If the information capacity of the resource pool is fixed, we should expect to see an inversely proportional relationship between set size and the amount of information retained per item (often measured with Fisher information, e.g., Bays, Catalao \& Husain, 2009; Oberauer \& Lin, 2017; van den Berg et al., 2015), as the fixed total resource is divided amongst the $\mathrm{N}$ items ${ }^{1}$. However, data from delayed reproduction tasks often fit extremely well to a power law relationship between set sizes and resources (Bays et al., 2009; Bays \& Husain, 2008; Oberauer \& Lin, 2017; van

\footnotetext{
${ }^{1}$ This model only applies to tasks containing multiple simultaneously presented memoranda, in which all items are equally salient and complex. When information are presented sequentially, we expect resources to be distributed unequally across memoranda, as indicated by sequence position effects.
} 
The conjunctive code model

den Berg et al., 2015). This is problematic for the assumption of a unitarily fixed capacity, because this power law model describes increasing resource capacity as a function of set size (Figure 2$)^{2}$. In other words, it appears as though additional resources becomes available when more items are encoded $-a$ finding that is at odds with the classic view of a constant WM capacity that is insensitive to change. This finding of a flexible WM capacity is not limited to the delayed reproduction task. For example, tasks measuring whether items are probabilistically stored in memory (Cowan, 2001; Pashler, 1988), have observed increasing capacity estimates with set size (Brady, Störmer \& Alvarez, 2015; Chen \& Li, 2017; Dai, et al., 2019; Endress \& Potter, 2014; Schurgin \& Brady, 2019), although the extent to which this occurs may depend on stimulus properties (Brady, Störmer \& Alvarez, 2015).

How can this apparent increase in memory capacity across set sizes be accommodated with the constraint of a constant capacity? Several possible explanations have been proposed. The power law relationship between set size and resource capacity was originally justified based on a model in which retrieval relies on maximum likelihood decoding of neural populations (Bays \& Husain, 2008; Schneegans et al., 2020; Taylor \& Bays, 2020). Under this perspective, the power law relationship emerges from correlations between the firing rates of neurons within the population, due to their overlapping tuning curves (Bays \& Husain, 2008; Seung \& Sompolinsky., 1993). This correlation means that when a single item is stored, there is redundancy, but dividing the same population between several items increases the amount of information capable of being stored, since the noise between neurons is less correlated (Averbeck et al., 2006). However, this view has been criticized as lacking theoretical justification, with alternative theoretically justified proposals for the effect of neural correlations on total WM capacity (Moreno-Bote et al., 2014; Oberauer \& Lin, 2017). Moreover the model assumes that neurons in a population can be dynamically re-allocated between different items in memory, which might be biologically unsubstantiated. In particular many models

\footnotetext{
${ }^{2}$ A power law model whose exponent equals 1 predicts a constant capacity across different set sizes. When the exponent is greater than 1 , it predicts decreasing capacity across set sizes. When the exponent is less than 1, it predicts that resource capacity increases set sizes. (e.g., Bays et al., 2009; Oberauer \& Lin, 2017; Ma et al., 2012).
} 
The conjunctive code model

(Schneegans \& Bays, 2018) require separate feature representations at each spatial location, which then entails carefully tuned cross-item rate normalisation to produce capacity effects.

Another line of explanation for increasing capacity with set size regards it as an optimal strategy (Sims, Jacobs \& Knill, 2012; van den Berg \& Ma, 2018). A resource-rational theory has been proposed in which the resource deployed to each item reflects an optimal trade-off between the behavioural benefit gained from drawing on additional resources to encode an item, and the neural (or effort) cost of deploying these extra resources. While this view is appealing because it provides a normative explanation of the observed set size effects, it is currently unclear what the mechanism might be that allows the brain to make this cost-benefit decision under the widely varying and changing contexts in which WM is used in everyday life. Furthermore, the results of studies manipulating motivation do not align with these predictions (van den Berg et al,. 2020).

One final perspective, which we explore in greater detail in this paper, is efficient compression of the studied information by extracting statistical regularities from the display (Brady, Kongle \& Alvarez, 2009; 2014; Schurgin \& Brady, 2019), or higher order information, such as ensemble statistics (Brady \& Alvarez, 2011), inter-item relationships (Bae \& Luck, 2017; Son et al., 2020), or configurations (Jiang et al., 2000). As more items are encoded, this higher order information becomes more readily available, potentially allowing greater compression as set sizes increase, and therefore boosting performance beyond that which would be expected if this compression were not taking place. There are now many experiments which show evidence for hierarchical representations, in which individual objects appear to be obligatorily grouped together into higher level clusters (Brady \& Alvarez, 2011; 2015; Corbett, 2017; Lew \& Vul, 2015; Orhan \& Jacobs, 2013). Brady \& Alvarez (2011) presented participants with memory displays of coloured circles of various sizes. When probed about the size of one of the circles, responses were biased towards the average size of the circles in that colour. Importantly, responses were biased towards the mean of items only in the same colour set, rather than the entire display, suggesting that the 
The conjunctive code model

circles of each colour were represented as belonging to distinct clusters. Another example of hierarchical representation in WM comes from evidence using Gestalt grouping cues (Corbett, 2017; Woodman, Vecera \& Luck, 2003). For example, in a change detection task, when one item in a probe display was cued, other items which could be grouped by proximity or connectedness received a boost in performance (Woodman, Vecera \& Luck, 2003). Son et al (2020) varied the similarity of oriented bars in a delayed reproduction task, allowing participants to encode the displays in terms of clusters of similar items. Task performance was determined predominantly by the number of clusters, rather than the number of items per se. An analysis of response errors showed that their participants were not simply remembering the cluster averages and forgetting individual orientations, because their responses were closer to the correct response than the mean of items in the cluster, albeit biased towards the cluster mean. Evidence of clustering effects have been shown in the domains of orientations (Son et al., 2020), size (Brady \& Alvarez, 2011), and location (Lew \& Vul, 2015; Jiang et al., 2000).

One issue with many of these models is that they are not necessarily embedded within our understanding of brain structure, and mathematical formalisations of these models are lacking. Therefore, we present a new model in which hierarchical information is abstracted from the study display and represented in composite codes in the visual system. How might these composite codes arise from the organisation of brain structure? Within the visual system, information has been proposed to flow anteriorly from V1 through two streams: a ventral 'what' pathway, and a dorsal 'where' pathway (Goodale \& Milner, 1992). As information flows through each stream, convergent connections allow the abstraction of increasingly complex features within widening receptive fields (Güçlü \& van Gerven, 2015). For example, areas V1 and V2 optimally respond to oriented bars presented at specific orientations and locations in the visual field (Hubel \& Wiesel, 1959), neurons in V4 respond to more complex features such as edge and shape (Roe et al., 2012), and cells in the inferior temporal cortex (IT) have large receptive fields which respond to a number of properties including shape (Gross, Rocha-Miranda \& Bender, 1972; Roa \& Ballard, 1999). 
The conjunctive code model

The sensory recruitment hypothesis (D’Esposito \& Postle, 2015; Pasternak \& Greenlee, 2005) states that WM maintenance utilises these visual processing areas, and that WM representation is simply a different state of activation of these systems which are otherwise used during perception. Single cell recordings in animal studies have found sustained activity during WM maintenance along all stages of the visual processing stream (Bergmann et al., 2016; Harrison \& Tong, 2009; Super et al, 2001; Xu \& Chun, 2006). Similarly, a number of de-coding studies have now shown that stimuli maintained in WM can be successfully decoded from relevant visual areas along the visual pathway (Christophel, Hebart, \& Haynes, 2012; Emrich, Riggall, Larocque, \& Postle, 2013; Harrison \& Tong, 2009; Lee, Kravitz, \& Baker, 2013; Riggall \& Postle, 2012; Serences, et al., 2009). This body of evidence described above supports the view that WM representation takes advantage of the preexisting representational hierarchy found in the visual cortex, with low level features represented posteriorly, and more complex- or abstracted features represented anteriorly. Furthermore, recent modelling work shows promising support that WM storage takes advantage of the existing hierarchy in the visual system (Alexander \& Brown, 2018; Hedayati, O'Donnell \& Wyble, 2021). If this is indeed the case, then an item in memory may be represented at multiple levels of abstraction in separate resource pools along the visual processing stream. Because the abstraction of low-level to higherlevel features is a highly automatic process during perception, information about displays of simple features such as simple oriented bars (or other simple features as are often used in WM experiments) may also be represented in these higher-level pools, such as pools for angles or corners during maintenance. For example, two nearby oriented bars may not only be encoded as orientations in primary visual cortex, but also as a composite code (such as the angular difference between them, or a corner) in extrastriate areas. As a result of this obligatory encoding of the relationships between items, the brain can take advantage of existing additional resource pools particularly as more items require maintenance. Figure 1 characterises our model, with multiple resource pools representing multiple levels of abstraction about the study display. In the model, this translates to greater performance than would be expected if only a single low-level feature pool was 
The conjunctive code model

used. One further assumption in our model is that the ability to form a composite code depends on the similarity between memoranda, with groupable items (such as being closer or more similar) being more likely to enter the same composite (e.g., Son et al., 2020), reflecting the map-like organisation of the visual cortex (Wandell, Dumoulin \& Brewer, 2007). If a composite code is formed from the composite of the target item and one of the non-targets (i.e. one of the items that was studied, but not probed), the participant might be able to use this relational information to guide retrieval of the low level feature. Performance can be boosted by integrating the direct read-out from the low-level feature store with the target value estimated from the composite code. As a result, the total amount of information that can be stored about the target (or capacity) increases with set size, because it can be offloaded to downstream stores representing its relationships to other items in memory.

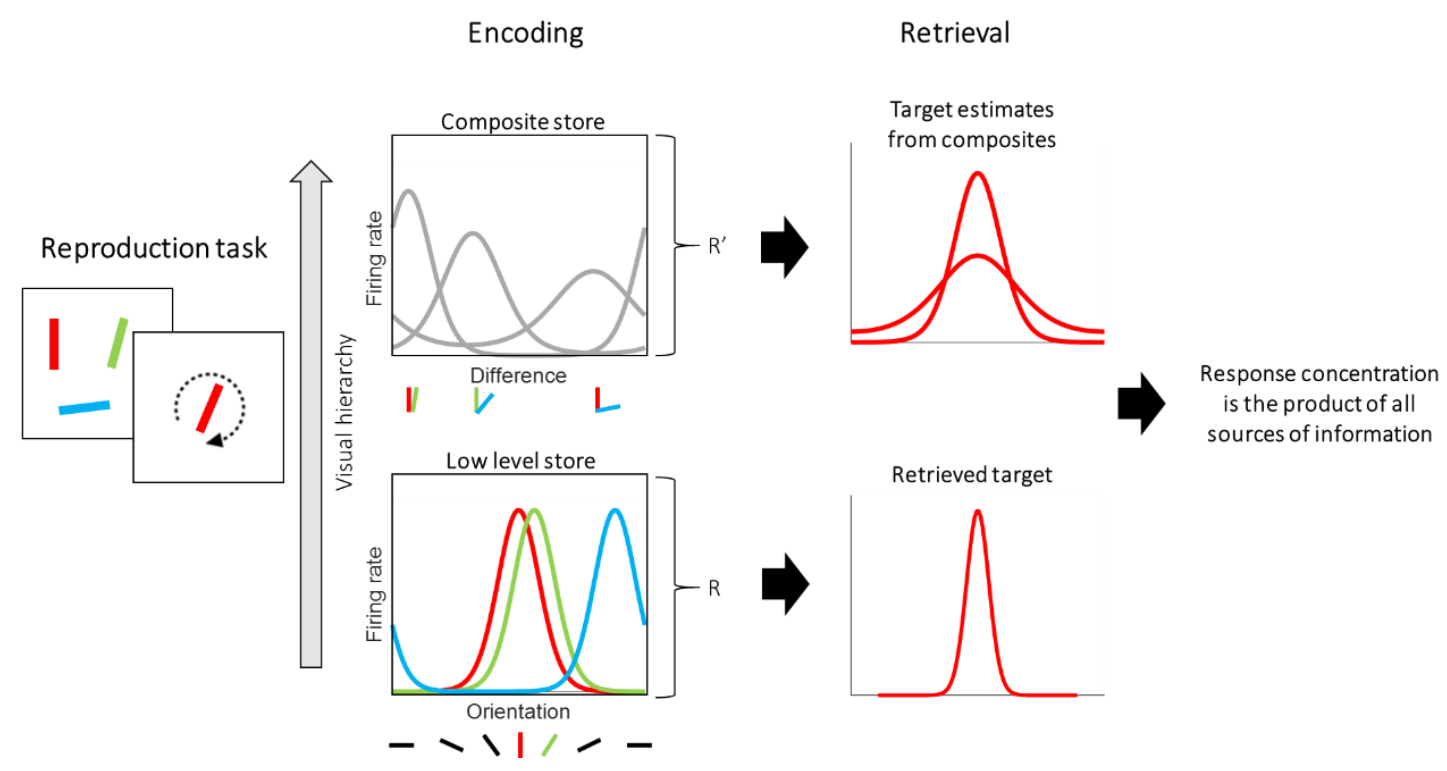

Figure 1. Characterisation of the composite code model.

In this example, despite the task only explicitly asking for participants to remember three orientations, they may obligatorily encode the relationships between them, stored in additional resource pools further down the visual processing stream. While our example includes only two pools 
The conjunctive code model

- simple orientations and corners, it is likely that the brain encodes visual information into multiple pools of increasing levels of abstraction.

\section{The composite code model formal description}

Our specific instantiation of this model draws influence from a number of existing models of continuous reproduction (e.g., Bays Catalao, \& Husain, 2009; Bays, 2014; Oberauer \& Lin, 2017; van den Berg et al., 2012; van den Berg et al., 2015; Zhang \& Luck, 2008). Here, we only model the case where all the items are encoded to avoid the issue that serial position will affect the amount of resource allocated to each item. We model WM as encoding the angles of studied memoranda (which in the general case may be any continuous primitive feature dimension, such as colour or orientation) using two pools of resource. The first, is a low-level pool which represents the veridical angle of the memoranda, with total resource R. The second, the conjunctive code pool represents the first-order relationships between the items, with total resource $\mathrm{R}^{\prime}$. At a broad conceptual level, there may be multiple resource pools, each representing more complex levels of representation (angles, corners, borders, shapes, faces etc.). At encoding, each item receives an equal share of the total resource in each pool:

$$
r=\frac{R}{N}
$$

(1)

With $R$ replaced with $R^{\prime}$, and $r$ with $r^{\prime}$, for the composite code pool. The quantity of resource allocated to each item determines the amount of noise in the representation, which we assume generates a normal distribution of errors around the correct value (for angles we use the circular analogue, the von Mises distribution). This assumption allows us to convert a quantity of information into the width of a distribution. From here on, the amount of resource $r$ or $r^{\prime}$ are converted to the precision of the representation, $\mathrm{K}_{\text {target }}$ or $\mathrm{K}^{\prime}$ respectively, by 
The conjunctive code model

$$
r=\frac{I_{1}(k)}{I_{0}(k)}-\ln I_{0}(k)
$$

(2)

where $I_{0}(k)$ is the modified Bessel function of order 0 , and $I_{1}(k)$ is the modified Bessel function of order 1.

At retrieval, the participant now has two sources of information about the target. The first source of information is the direct read-out of the low-level feature pool. The second source of information is an estimate of the low-level target feature value by integrating evidence about the identity of each composite with the feature value of each respective non-target. Because we do not model systematic drift or repulsive or attractive bias, the mean of the estimate distribution is zero. Because estimating the target from the composite pool involves two sources of noise - the noise in the non-target representation and the noise in the composite representation, an approximation of the variance of this estimate is the sum of the variance of those two distributions:

$$
\frac{1}{\kappa_{e s t}}=\frac{1}{\kappa}+\frac{1}{\kappa^{\prime}}
$$

(3)

Finally, Bayesian inference combines the direct read-out with the estimates from all available composites, to produce a posterior estimate of the target orientation. Given the way differences are encoded in visual cortex, we tested two possible constraints. In one model (similarity-based composite codes) a pair of items are more likely to be combined into a composite code if they are more similar to one-another. This can be modelled by scaling the strength of the facilitation by the angular difference, $\Delta \mu$, between items. The response concentration for this model is

$$
K_{i}=\kappa_{i}+\sum_{j=1}^{m} \kappa_{e s t}^{j} e^{-\epsilon\left|\Delta \mu_{j}\right|}
$$

(4) 
The conjunctive code model

The degree to which each target estimate, $\mathrm{j}$, influences the posterior depends on the absolute angular difference between the target and non-targets $(|\Delta \mu|)$, weighted by $\varepsilon$. This corresponds to the effect that higher-order composite codes will only be created, and ultimately influence retrieval, if the two items are sufficiently similar enough that they fall within the composite code's receptive field.

The second alternative we tested is one in which the probability of a composite being encoded depends on the spatial proximity between two stimuli:

$$
K_{i}=\kappa_{i}+\sum_{j=1}^{m} \kappa_{e s t}^{j} e^{-\lambda d_{j}}
$$

(5)

In which d is the Euclidean distance from the target's position to the position of each non-target, weighted by the receptive field size $\lambda$.

We compared these two conjunctive code models to one in which only a single store is used to represent the features themselves. The response concentration for the target under this model is given simply by equation 2 , and equations $3-5$ can be ignored.

The tasks we are modelling also require encoding the binding between features, and there are multiple ways in which $\mathrm{N}$ items can be (correctly and incorrectly) bound ${ }^{3}$. For example, encoding a vertical red bar and a horizontal blue bar requires correctly remembering that the vertical bar was red and not blue - i.e. one of two alternatives. Therefore, storing the bindings of features to objects itself requires information capacity. Ultimately the amount of stored binding information determines the probabilities with which a subject recalls the target (correct) and non-target (incorrect) features, so larger set sizes will require more binding information. We assume this taps a separate resource to

\footnotetext{
${ }^{3}$ In the case of remembering two feature dimensions, the maximum number of possible bindings is $\mathrm{N}$ ! if bindings are mutually exclusive (for example, remembering that the vertical bar is red means that the horizontal bar will not be erroneously remembered as red). If they are not mutually exclusive, the number of possible bindings is $\mathrm{N}^{2}$ (for example erroneously remembering that both bars were red).
} 
The conjunctive code model

the features themselves. The amount of binding information theoretically available for each item, $r_{\text {binding }}$ was calculated by dividing a fixed binding resource, $R_{\text {binding }}$ by $N$ items. The probability of correctly recalling each of the items in the display was calculated as

$$
\begin{gathered}
r_{\text {binding }}=\log N+\sum_{i=1}^{N} p_{i} \log p_{i} \\
=\log N-p_{\text {target }} \log p_{\text {target }}-\left(1-p_{\text {target }}\right) \log \frac{1-p_{\text {target }}}{N-1}
\end{gathered}
$$

(6)

Where $p_{i}$ is the probability of recalling the target, and $p_{2: N}$ is the probability of erroneously recalling each of the non-targets $\left(\frac{1-p_{i}}{N-1}\right)$ instead.

Putting all of these together, the probability of the participants response in radians, $\theta$, on a particular trial, is a weighted mixture of distributions for of each presented items, with probability $p_{i}$, and concentration $K_{i}$.

$$
p(\theta)=\sum_{i}^{m} V M\left(\theta-\theta, K_{i}\right) p_{i}
$$

(7)

$\theta$ is the studied feature value in radians. VM is the von-Mises density function

$$
V M(\kappa)=\frac{e^{\kappa \cos (\theta-\theta)}}{2 \pi I_{0}(\kappa)}
$$

(8)

\section{Method.}

We conducted a delayed reproduction task for orientation, varying the set size. The aim of this experiment were two-fold. First, to provide an additional data set for analysis with a relatively large number of trials (100) per condition. Second, several models, including ours, predict an increase in total memory capacity with increasing memory set size. Therefore, to test this relationship, we 
The conjunctive code model

extended the set size beyond that which is usually tested in the literature. Specifically, we tested set sizes within the range 2-14 items per display. Does this steadily increasing relationship hold true, or would we see a plateau at larger set sizes?

\section{Participants}

Ten medical science students from the University of Oxford participated in the experiment (Ages 19$28, \mathrm{MF}$ ratio $=1: 1$ ). All participants reported normal- or corrected-to-normal vision. They gave written informed consent prior to the start of the experiment and received $£ 30$ as financial compensation for participating. Ethical approval was granted by the University of Oxford's Medical Sciences Research Ethics Committee.

\section{Stimuli}

Stimuli were displayed on a 24-inch LCD computer displayed at a viewing distance of $60 \mathrm{~cm}$. The experiment was programmed in Psychopy builder (Pierce et al., 2019). The display was presented on a black background. The study consisted of a variable number of oriented bars (length $=4 \mathrm{~cm}$, width $=0.5 \mathrm{~cm}$ ) presented on an imaginary circle (radius $=10 \mathrm{~cm}$ ) at the centre of the screen. The colours of the bars were chosen from 15 evenly spaced values in the cieLab colour space $\left(L=70, a_{\text {centre }}=25\right.$, $b_{\text {centre }}=20$, radius $=90$ )

\section{Procedure}

Each trial began with a brief blank interval (100ms), and the message "Get ready!" (300ms). After an additional $100 \mathrm{~ms}$, the study display was presented, consisting of random number of simultaneously presented oriented bars (set sizes $=2,3,5,8,10,12,14$ ), and was displayed for 1 s per item present. 
The conjunctive code model

The study display was followed by a brief blank interval $(250 \mathrm{~ms})$, a mask $(50 \mathrm{~ms})$ and another blank interval (250ms). Next, the probe display was presented, in which one of the oriented bars reappeared in its original colour and location, but in a random orientation. The participants were instructed to use the mouse to re-orient the bar back to its original orientation and confirm their response by pressing the space bar. They were instructed to take their time to respond and favour accuracy over response times. They could take as much time as they preferred to respond, but after 4s a prompt appeared in text reminding them how to respond and to press the space bar. Finally, after they entered their response, both the correct response and their selected response were presented for $2 \mathrm{~s}$ (with $50 \%$ transparency) to provide feedback. The experiment was conducted across two testing sessions. Each session consisted of 25 blocks of trials, each containing 2 repetitions of each condition in a random order. In total, each participant did 100 trials of each set size. Across both sessions, the experiment took approximately three hours per participant. Each session began with a practice block consisting of one repetition of all conditions, with additional text instructions explaining the task.

\section{Results}

In addition to analysing data from this task, we also acquired continuous reproduction data from four previously published data sets available online. Circular reproduction data allows straightforward model fitting, as data in this task typically follows a mixture of von-Mises distributions, for which analytical measures of entropy are readily available. However, other tasks can be analysed in a similar way (for example by measuring the entropy in tasks which can be modelled with the normal distribution). The details of each experiment we analysed are presented in table one. We did not include set sizes of 1 , where relevant, in model fitting, because WM models often over-predict precision for this condition; this may be because they do not account for motor and perceptual noise that become the limiting factor when memory itself is very precise. In the next sections, we discuss 
The conjunctive code model

several predictions made by the composite code model and compare these against the predictions of a comparable model without composites. Additionally we re-analyse existing open data-sets in the literature to test specific predictions of the composite code model. Model predictions were made by averaging the results of 10,000 simulations per condition for each set of fitted parameters (we estimated one set of fitted parameters per participant).

Table one. Details of the experiment data analysed.

\begin{tabular}{cccc}
\hline Paper & Report feature & Cue feature & Set sizes \\
\hline Bays et al (2009) & Colour & Location & $1,2,4,6$ \\
Gorgoraptis et al (2011) & Orientation & Location + Colour & $2-5$ \\
Oberauer \& Lin (2017) & Colour & Location & $1-8$ \\
Souza et al (2014) & Colour & Location & $2-8$ \\
Our data & Orientation & Location + Colour & $2,3,5,8,10,12,14$ \\
\hline
\end{tabular}

This paper was motivated by the observation that WM capacity appears to increase across set sizes. Before fitting the model, we first calculated the quantity of resource per item as Shannon information (Shannon, 1948), which is a measure of the number of bits required to maintain each item with that level of precision. For circular data, this is calculated with

$$
\log (2 \pi)+\sum_{i=1}^{N} p_{i} \log p_{i}
$$

(9)

where $p_{i}$ is the probability of the response error in radians in each bin (we used 100 bins). We then calculated a measure of total information capacity at each set size by multiplying the Shannon 
information per item by the set size (Figure 2). Note that the slope for information-per-item is shallower than expected for a fixed capacity system, and total resource increases across set sizes.
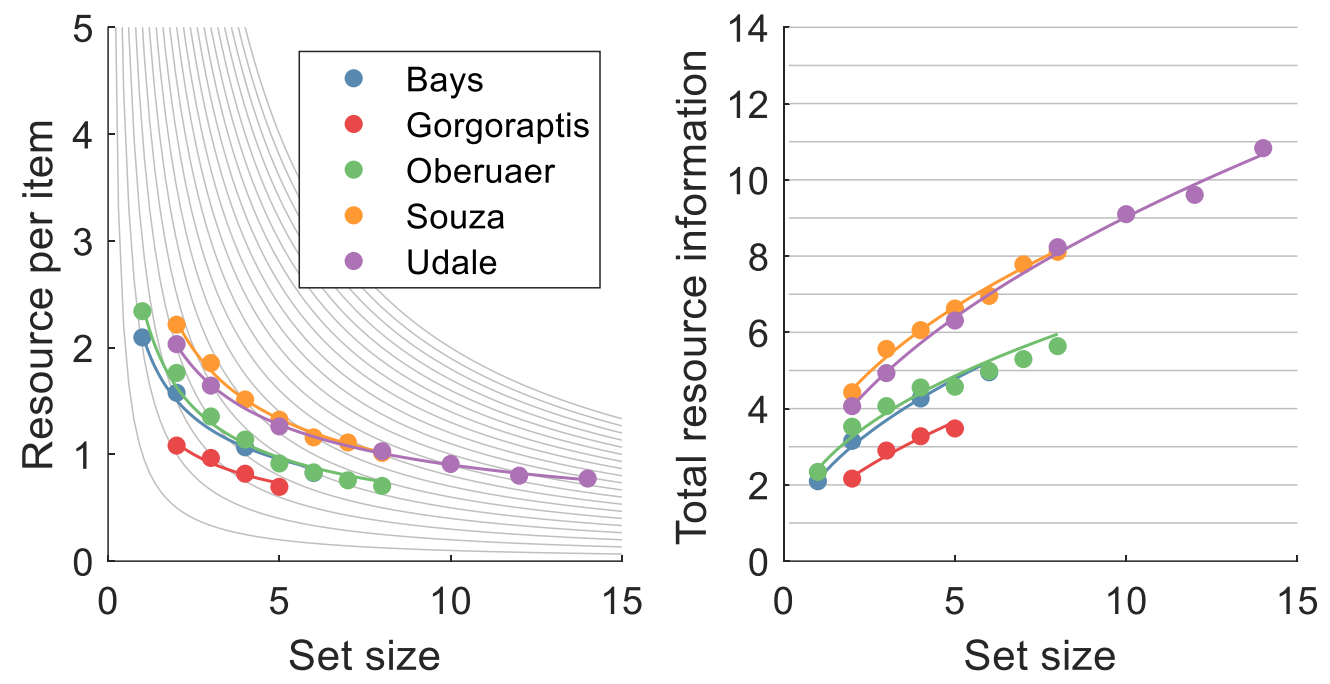

Figure 2. Left: Resource (Shannon information) per item for each set size. Right: Total resource maintained at each set size.

Note. Grey lines represent the curves expected if capacity remained fixed across set sizes, for different values of memory capacity.

We fitted the three models (null no composite, similarity-based composites, and proximitybased composites) Two datasets did not include stimuli location data, and therefore we did not fit the proximity-based composite code model to these datasets. Table two shows the differences in AIC (Akaike, 1974) for the three models we compared across the five experiments. Both composite code models performed much better than the null model, with the similarity-based composite code model performing much better than the proximity-based composite code model. 
The conjunctive code model

Table two. Model comparison using $\triangle \mathrm{AIC}$.

\begin{tabular}{cccc}
\hline Paper & Null model & $\begin{array}{c}\text { Similarity-based } \\
\text { composites }\end{array}$ & $\begin{array}{c}\text { Proximity-based } \\
\text { composites }\end{array}$ \\
\hline Bays et al (2009) & 529.72 & 0 & N/A \\
Gorgoraptis et al (2011) & 26.1 & 0 & 18.96 \\
Oberauer \& Lin (2017) & 809.22 & 0 & 43.64 \\
Souza et al (2014) & 748 & 0 & N/A \\
Our data & 971.27 & 0 & 153.09 \\
\hline
\end{tabular}

$\triangle \mathrm{AIC}$ is the difference in AIC values for each model from the AIC value of the best fitting model. A

value of 0 indicates the best fitting model, with larger values representing less parsimonious fits than the best model.

Figure 3 shows the effect of set size on mean absolute error in the delayed reproduction task for five experiments. Mean absolute error increases with set size. The composite code model (Figure 3, solid lines) captured the slope better than a simple single-store model without composite codes (Figure 3, dashed lines) which showed a much steeper increase in error across set sizes. This analysis indicates increasing effective capacity with set size. 

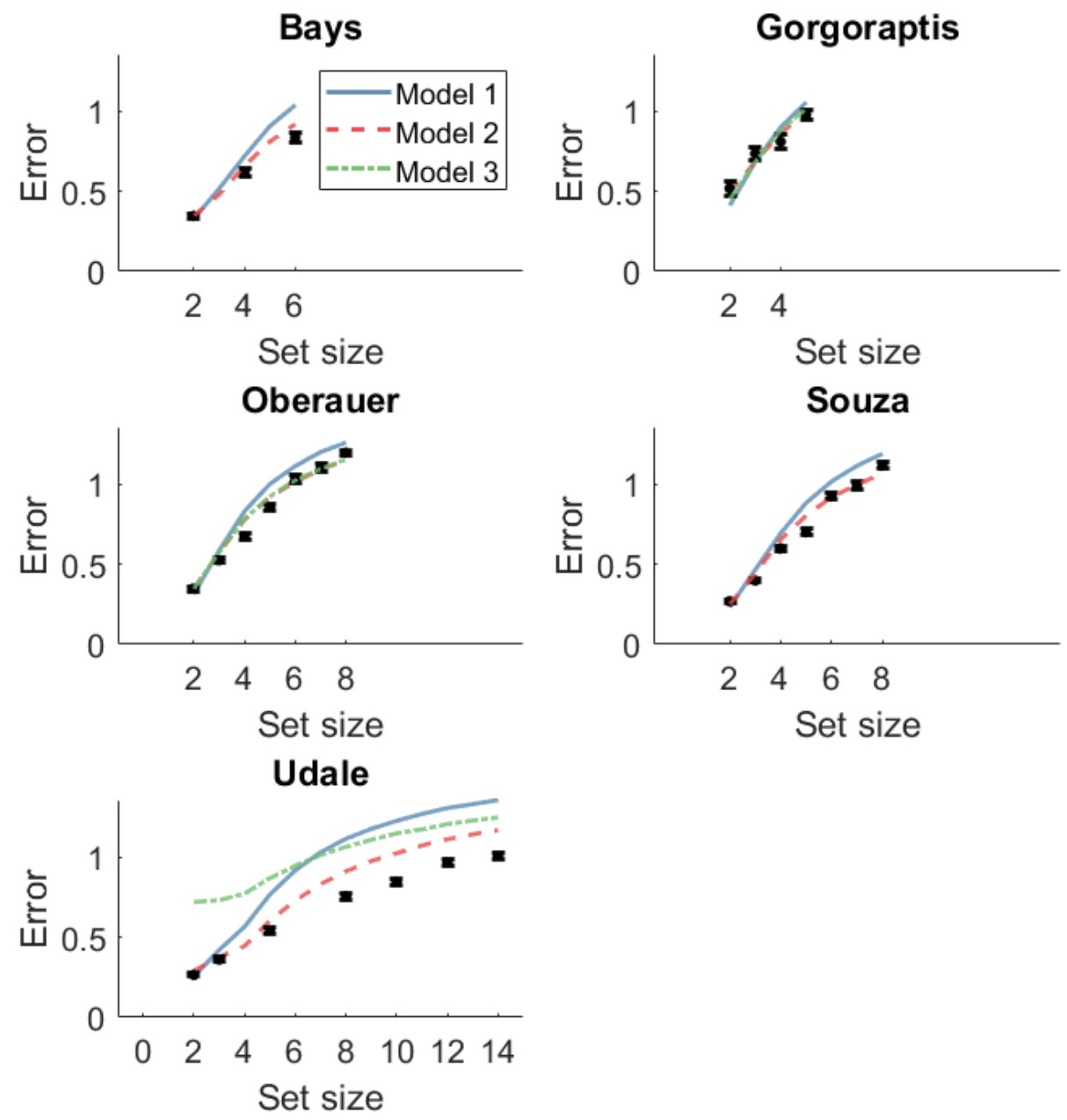

Figure 3. Set size effect on mean absolute error.

Dots represent the means across conditions, with error bars representing the standard error of the mean. Solid lines represent the predictions made by the composite code model. Dashed lines represent predictions made by a simple single-store model.

In continuous reproduction tasks, participants must maintain two types of information: the set of features present, and the bindings between those features and their cues (e.g., which colour appeared at which location). Next, we separately quantified the total feature information (equation 10) and total binding information (equation 11) stored across set sizes, for both the observed data 
The conjunctive code model

and simulated data from our two models. Using the analytic formula for Shannon information contained in the von-Mises distribution, the total amount of information maintained about the retrieval features is estimated with:

$$
I_{\text {feature }}=N\left(\kappa\left(\frac{I_{1}(\kappa)}{I_{0}(\kappa)}\right)-\ln I_{0}(\kappa)\right)
$$

where $\kappa$ is the concentration of the von-Mises distribution fitted to the distribution of responses around the target, and $N$ is the number of items encoded. The estimated Shannon information for each item is multiplied by $\mathrm{N}$ to give us an estimate of the total information stored. The total quantity of information maintained about the feature bindings is measured with:

$$
I_{\text {binding }}=N\left(\log N+\sum_{i=1}^{N} p_{i} \log p_{i}\right)
$$

where $p_{i}$ is the probability of recalling of each item, $i$, in the memory set, which is in turn calculated from the mixture model's fitted misbinding parameter $\beta$. Specifically, $p_{1}=\beta$, and $p_{2: N}\left(\frac{1-p_{i}}{N-1}\right)$. These parameters, kappa and beta, are estimated using a two-component mixture model in which participants either correctly recall the probed target, or erroneously recall a studied item which was not probed ('non-targets'). Under this model, the probability of responding with the reported orientation $\theta$ in response to target item $\theta$ or non-target items $\theta_{i}$ (in radians) is:

$$
p(\theta)=(1-\beta) V M(\kappa, \theta-\theta)+\beta \frac{1}{N-1} \sum_{i}^{m} V M\left(\kappa, \theta-\theta_{i}\right)
$$

$V M\left(\kappa, \theta-\theta_{i}\right)$ denotes the probability density function (PDF) of the von Mises, with concentration parameter $\kappa$. 
The conjunctive code model

We found that total feature information increases with set size in the data, which was tracked well by the composite code model (Figure 4, red/green dashed lines), whereas the model without composite codes predicted a fixed limit on total feature information (Figure 4, blue solid line). All three models assumed a limited capacity for binding (Figure 5), which tracked the data well, with the exception of our dataset which, remarkably, showed increasing binding capacity across set sizes.
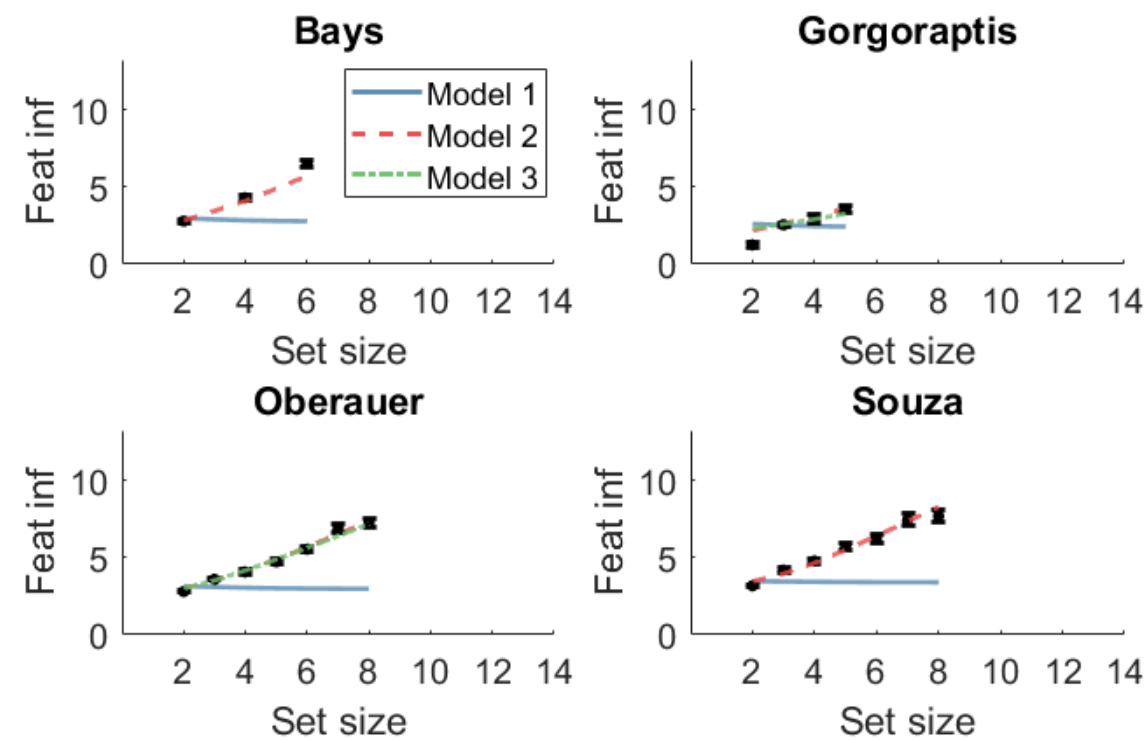

Souza

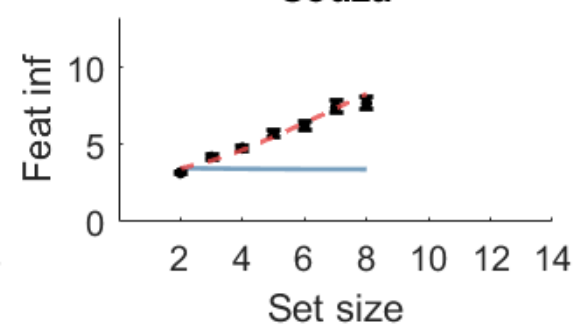

Udale

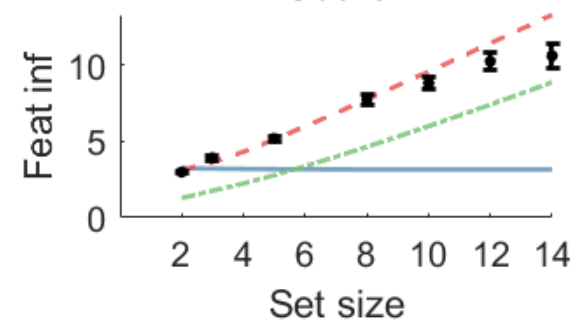

Figure 4. Total feature information across set sizes.

Dots represent the observed means, with error bars representing the standard error of the mean.

Solid lines reflect predictions from the composite code model, and dashed lines represent predictions 
from a simple single-store model. Feature information was measured from the modelled width of response distributions, for each set size.
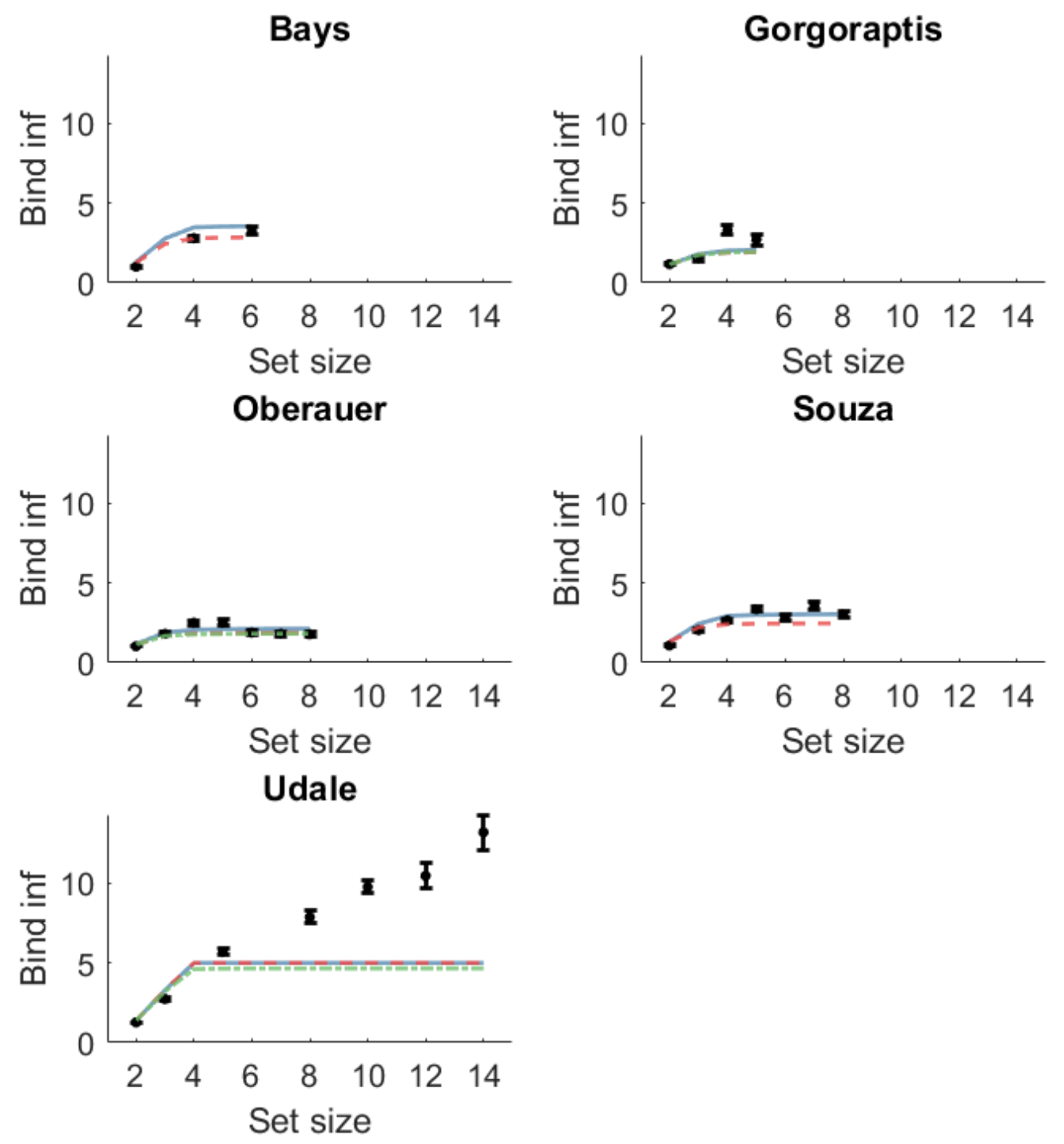

Figure 5. Total binding information across set sizes.

Dots represent the observed means, with error bars representing the standard error of the mean.

Solid lines reflect predictions from the composite code model, and dashed lines represent predictions 
The conjunctive code model

from a simple model without composite codes. Binding information was computed from the modelled probability of retrieving the correct and incorrect memory items.

One further prediction of the similarity-based composite code model is a similarity benefit an effect often found in the literature (Oberauer \& Lin, 2017; Lin \& Luck, 2009; Zhang et al., 2016). This is predicted by the composite code model because displays containing features that are on average more similar, will be more likely to encode composites, and therefore receive a performance benefit. We measured the effect of mean display similarity on mean task performance, split into 5 bins, and compared this to the predictions from each model. For comparison we have plotted the data with the predictions of the similarity-based composite code model (Figure 6) and the null model (Figure 7) separately.
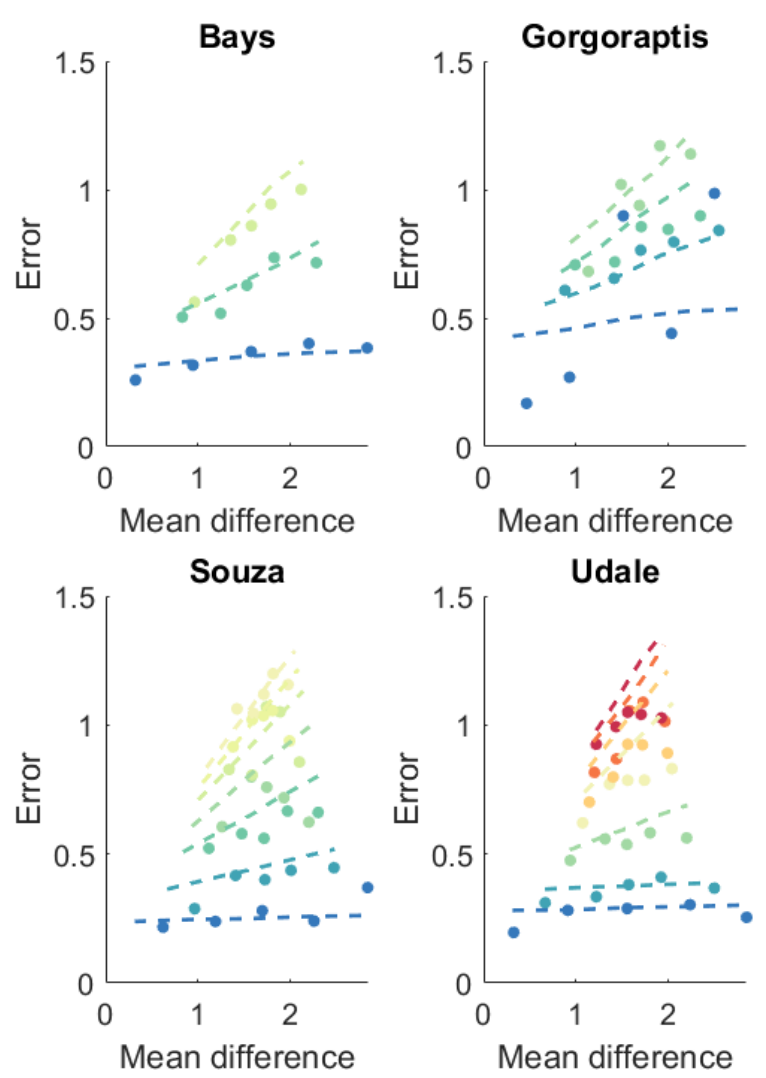
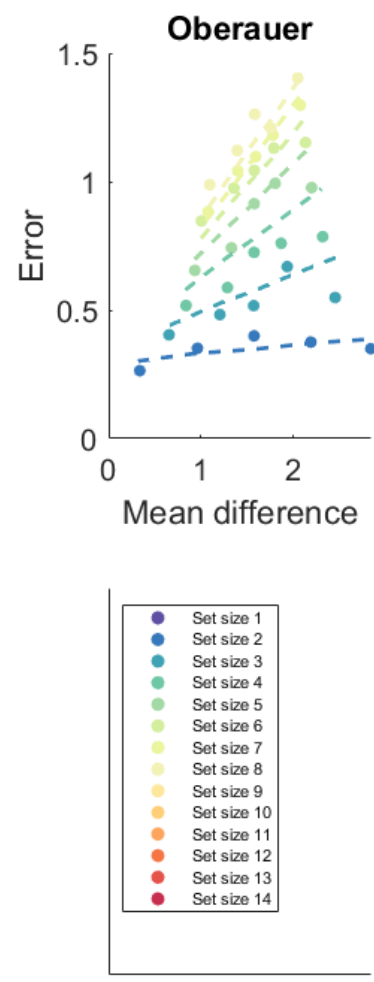
The conjunctive code model

Figure 6. Mean absolute error as a function of mean display difference with composite code model overlay.

Dots represent the observed data, split into 5 bins. Lines represent predictions from the null model.
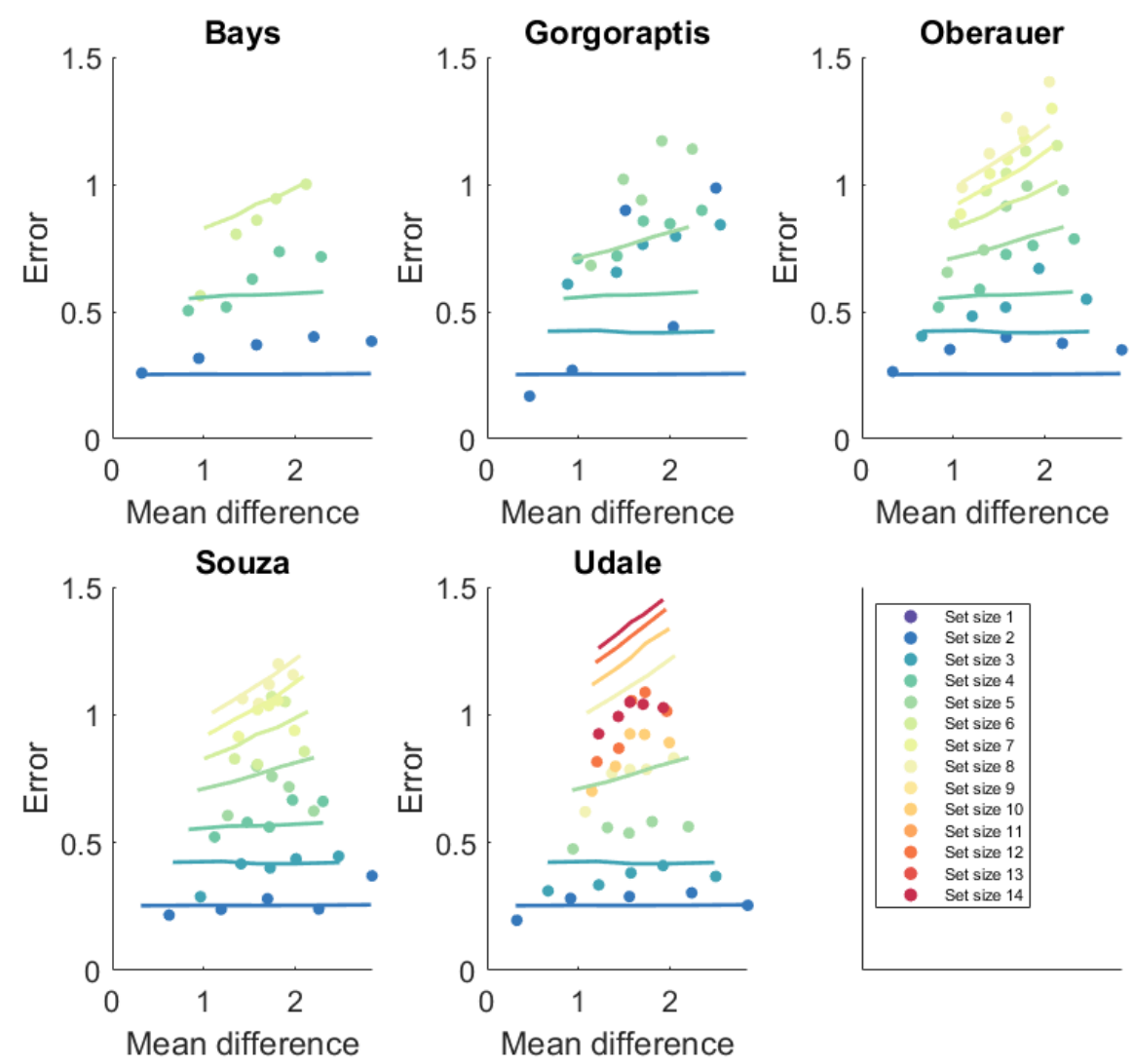

Figure 7. Mean absolute error as a function of mean display difference with null model overlay.

Dots represent the observed data, split into 5 bins. Lines represent predictions from the null model.

We found reliable effects of similarity across all five experiments: Displays containing more similar items (lower average mean difference) led to lower error, particularly at higher set sizes. These effects map quite well on to the predictions of the composite code model. However, these effects can also be explained relatively well by swap errors: if the feature values in a display are 
The conjunctive code model

similar and a swap error is made, the error between the response and the target value will be small. In contrast, confusing two very different items will lead to a much larger error. This explanation is supported by the consistent fits of the null model which predicts that the benefit from similarity comes solely from swap errors (Figure 7).

Total information increases for simultaneously- and sequentially- displayed stimuli.

The benefit from composites may depend on the opportunity to encode items in relation to other items. Comparing sequential and simultaneous encoding could provide a test of this possibility. Gorgoraptis et al (2011) conducted a delayed reproduction experiment in which participants viewed multiple memoranda presented either simultaneously or sequentially. We found that while both types of information increased with set size, a shallower slope was observed for sequentially presented information (Figure 8). This suggests that, if the increase in information results from composite codes, the composites can form both during perception and within memory. By comparison, a simple single-store model without composites would predict a slope of 0 .

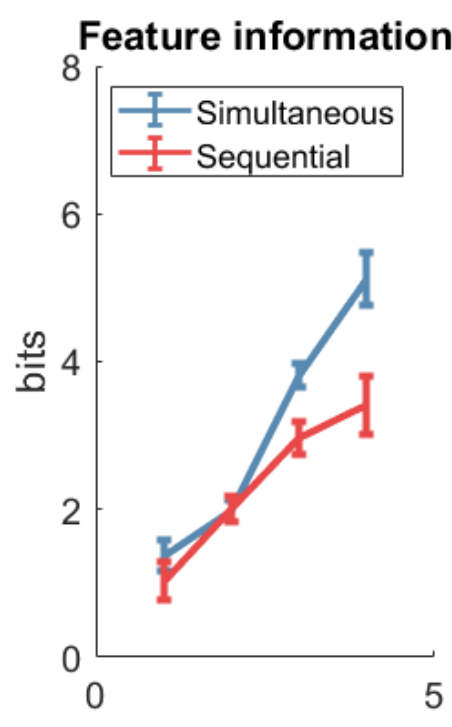

Set size

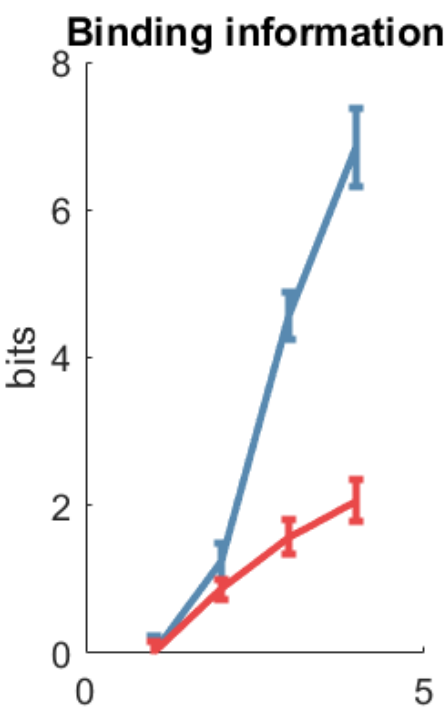

Set size 
The conjunctive code model

Figure 8. Total empirical information for sequential and simultaneous displays (Gorgoraptis et al., 2011).

Error bars reflect 95\% within subjects confidence intervals.

Error correlations are consistent with composite coding

, A novel prediction of the composites code model is that responses for items within the same trial should be correlated. This is because any drift in the representation of one of the composites should have the same effect on retrieval of both of its associated low-level features. If the composite code widens, responses should drift away from the correct response. If the composite code narrows, responses for all items should be attracted towards the correct response. Tabi et al (in preparation) recently conducted a whole report delayed reproduction task for three locations

(Figure 9, top) or colours (Figure 9, bottom). In that dataset we measured the correlation between each of the retrieved items. We removed trials where any of the absolute response error was greater than 1 standard deviation from 0 to filter out most of the non-target or uniform responses. We also filtered out trials where the difference between the memoranda were greater than $\pi / 2$ radians because we expect the effect will only occur for similar stimuli (when composites are likely to be encoded). After filtering, we calculated the mean error for each item, split into 10 bins. We found positive correlations for both location and colour retrieval. The correlation coefficients were strongest for successively retrieved items (items 1-2, or 2-3, but not items 1-3), in keeping with a role of sequential contrasts at encoding for forming composite codes (Note that although recall order was free, participants reported items in the same order they were presented on $90 \%$ of trials). Additionally, we acquired a similar dataset from Peteres et al., (2019) and conducted the same analysis. Here we found small positive correlations ( $r=0.59)$, but these were not statistically significant (Figures 10-11). 

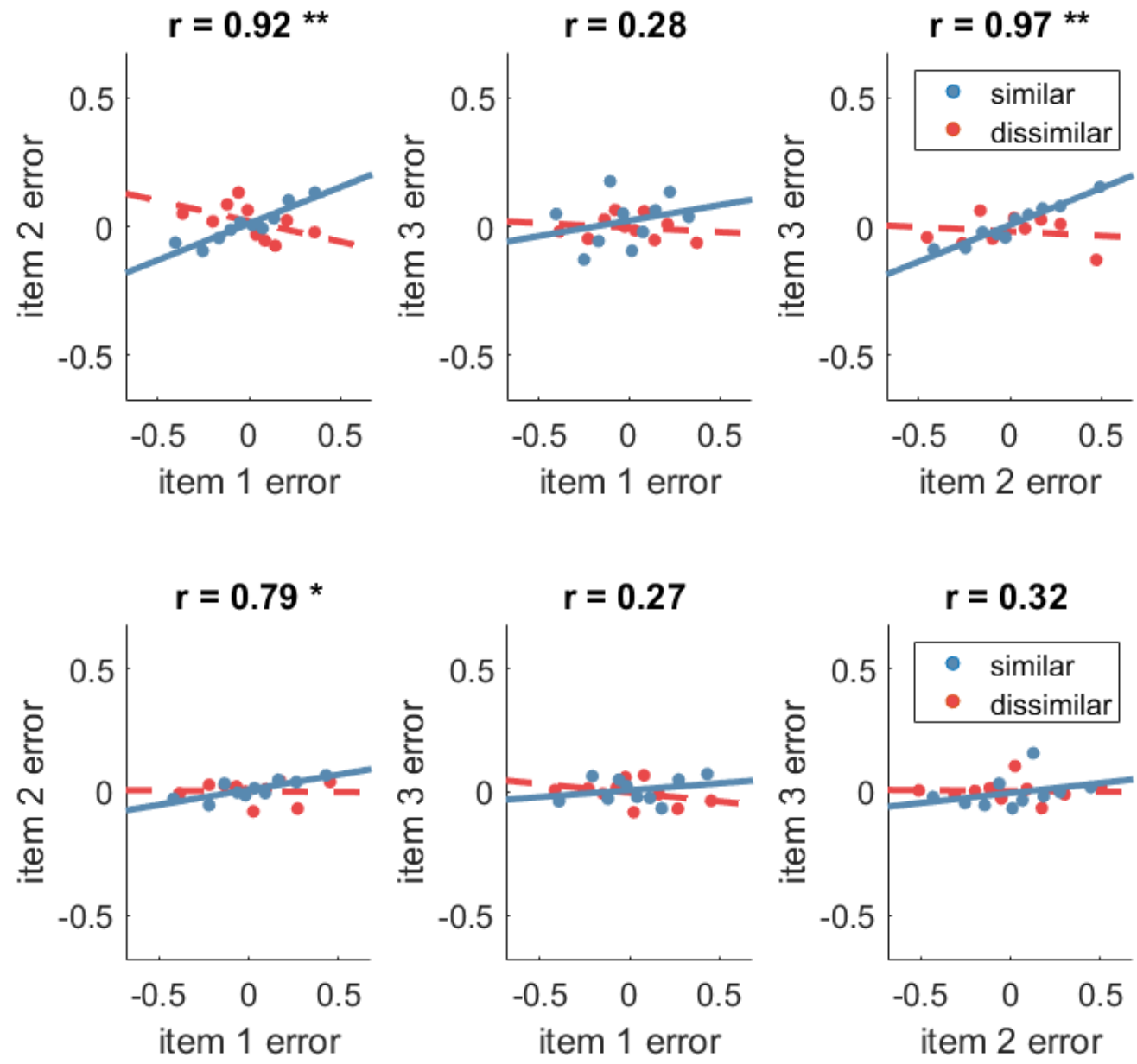

Figure 9. Positively correlated responses from items within the same display.

Top: Location memory. Bottom: Colour memory. Data are split according to whether the two items were similar (a difference less than $\frac{\pi}{2}$ radians, blue solid lines) or dissimilar (a difference greater than $\frac{\pi}{2}$ radians, red dashed lines). $*$ Indicates a significant correlation with a $p$ value below .01 , and ${ }^{* *}$ indicates a significant correlation below .001 . 
The conjunctive code model

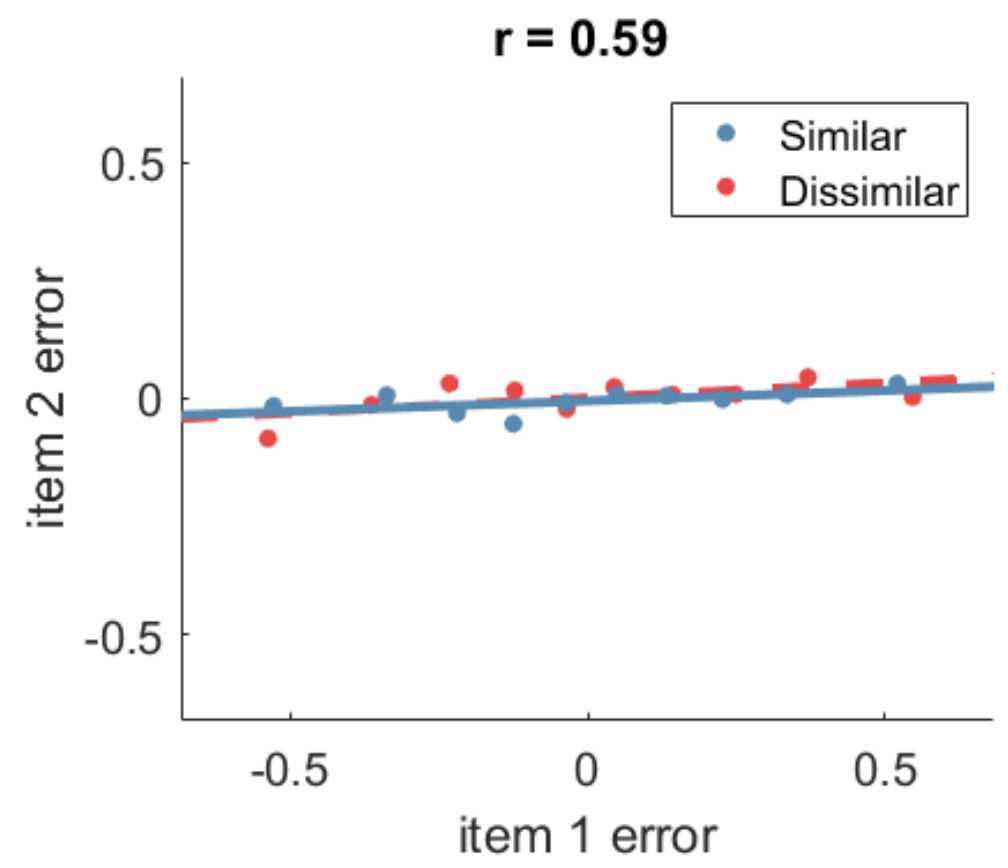

Figure 10. Response error correlation between items in whole report probe for set size 2 (Peters et al., 2019). 

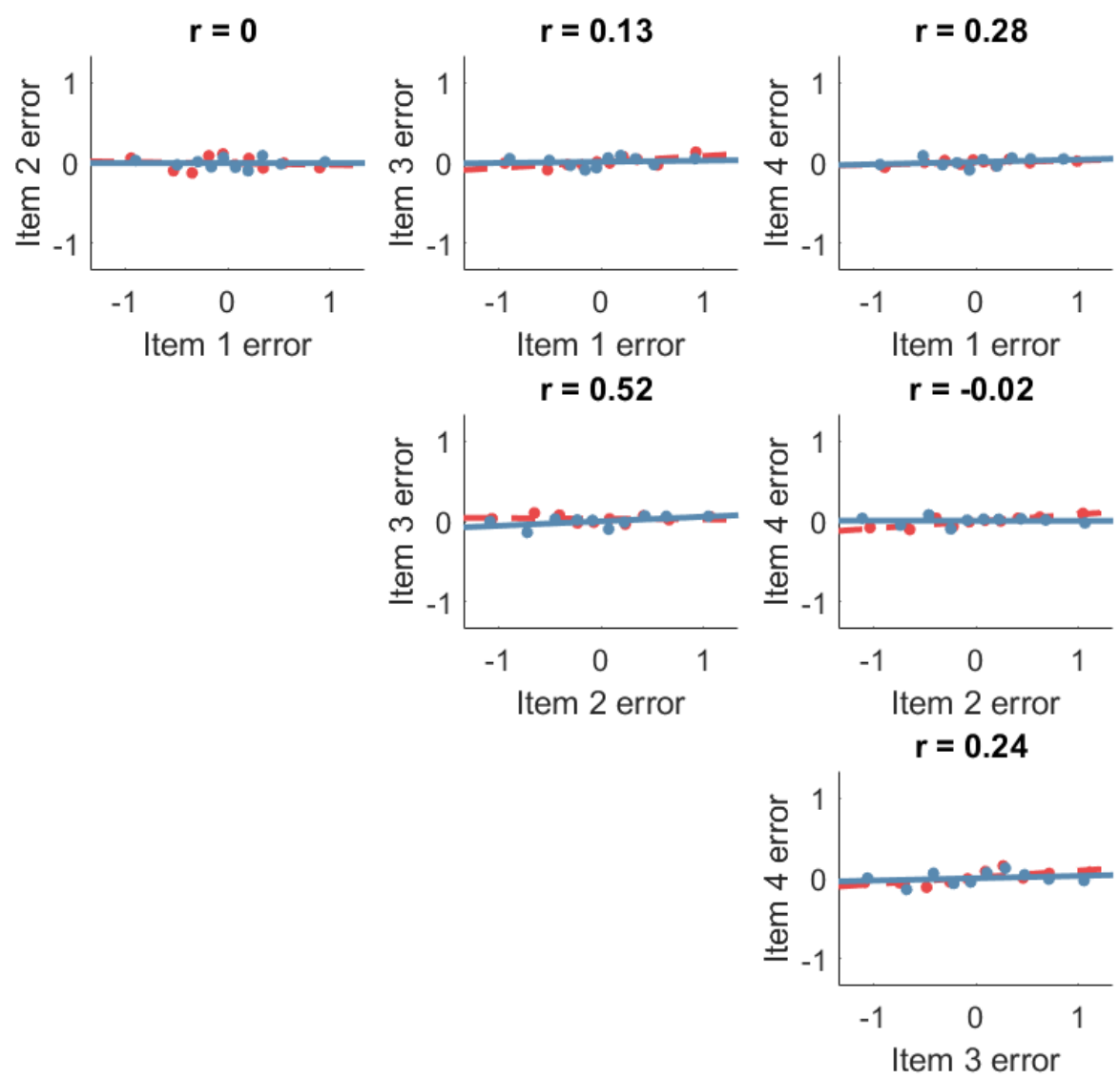

Figure 11. Response error correlation between items in whole report probe for set size 4 (Peters et al., 2019).

Data are split into similar and dissimilar displays. $\mathrm{R}$ values represent the correlation coefficient for the similar items (blue).

\section{General discussion}

The motivation for this paper was to propose an explanation for the common observation that more information is stored in WM at larger set sizes in WM tasks. Three main views compete to explain this effect. The first is that correlated noise in the neural populations used to represent WM contents leads to redundancies for single items, that can be utilised with larger set sizes items (e.g., Schneegans et al., 2020). The second view is that additional resources are available to be drawn 
The conjunctive code model

upon if needed, with the quantity of resource deployed depending on an optimal trade-off between the behavioural benefit of additional encoding against the cost of this additional effort (van den Berg \& Ma, 2018). Finally, the third view is that higher order information (which is more readily available when more items are encoded) can be extracted and encoded about the display in a separate store, which benefits performance at retrieval (e.g., Brady \& Alvarez, 2011). Under this perspective, there already exist a number of candidates for exactly what type of higher order information is encoded, such as ensemble statistics (Brady \& Alvarez, 2011), inter-item relationships (Bae \& Luck, 2017; Son et al., 2020), or configurations (Jiang et al., 2000). We quantitatively simulated a model in which composite codes are represented: representations of the differences between individual items. This view is motivated by the known organisation of the visual system in which low-level features, such as orientations, converge to form composites, such as a corner formed of two orientations. This model assumes that in tasks which require maintenance of low level features (widely used in the visual WM literature), higher order composites are obligatorily encoded and subsequently used to benefit performance.

In this paper, we compared the fits of three models. The first was a straightforward single store model which only encoded the feature values in the task. Models 2-3 both stored composite codes, albeit making different assumptions. The first composite code model was a similarity-based composite code, in which more similar items have a greater likelihood of generating a composite representation. The other was a proximity-based composite code model, in which items close in spatial proximity had a greater likelihood of forming a composite. Both composite code models performed very well at predicting the data, and measures of parsimony (AIC) suggest that they outperform the null model. Of all three models, the similarity-based composites model performed the best, both in terms of AIC, and in posterior predictive checks against the data.

We draw on a number of sources of evidence in support of the similarity-based composite model. First, the model highly predicts the set size effect in which performance is poorer at larger 
The conjunctive code model

set sizes. Second, when we measure the total information capacity for this model, it tracks extremely well with the independently measured total information from behavioural data, which increases with set size. The model also tracked very well the capacity for binding information, which unlike feature information, appears to be asymptotic in most cases. However, it was not necessary to invoke composite codes to explain the relationship between set size and binding capacity, which was predicted well by a limited binding resource mechanism.

The third source of evidence in support for the composite code model was the close tracking between data and predictions for the relationship between the similarity between study items and memory performance. However, the caveat should be noted that the null model also predicted the similarity benefit (but did show poorer fit according to AIC), which appears to be largely explained by swap errors (see also Oberauer \& Lin, 2017).

The fourth piece of evidence in support of composite codes is that the increase across set sizes in total information for simultaneously encoded displays is higher than that for sequentially encoded displays. This suggests that composites are encoded both during visual perception and during memory maintenance to a certain extent. In the case of simultaneous displays, the composites can be encoded during both the study display and later during maintenance. However, in the case of sequentially encoded displays, relationships between items can only be encoded during maintenance. As a result, the slope of the increasing capacity across set sizes is lower in the sequential condition than in the sequential condition.

The final piece of evidence in support of composite codes is that the errors in whole report tasks, in which all items from a display are recalled, are correlated. The model predicts correlated errors within the same display because the composite is used to retrieval the low-level feature values. If any drift occurs in the composite code, such as a widening or shrinking, this information will feedback to the low-level features. For example, if the composite representation widens, the low-level responses will be biased away from each other. We found this in a re-analysis of data from 
The conjunctive code model

our own lab (Figure 9), supporting the model. We also found weak positive correlations in an analysis of data from another lab, although these were not statistically significant. Correlated responses and biases in delayed reproduction is often difficult to detect, and it is possible that the correlations are too weak to detect reliably. Notably these were strongest for consecutive items, in line with a role of simultaneous contrast. Relatedly, similarity-dependent correlations between recall errors have also been found across trials (Bae \& Luck, 2017), suggesting that composites may also be formed from residual activation of previously maintained items in memory.

The composite code model makes a number of predictions which we have not been able to test, but for which there is evidence in the extant literature. One prediction made by the composite code model is that we should see greater brain activation at larger set sizes during WM maintenance - particularly when the items in the study display are more similar to one-another. For example, we should see a spreading of activation in fMRI (functional magnetic resonance imaging) BOLD (blood oxygen level dependent) signals as additional regions are activated to encode composite representations. Indeed, BOLD signals often increase in the visual cortex across set sizes (Christophel et al., 2012; Pessoa et al., 2002), although a widening of active regions has not been reported to our knowledge. At face value, this is against the predictions of a population normalisation-based view of capacity limits [Bays].

Similarly, the contralateral delay activity (CDA), which is a slow-wave negativity that tracks memory load during maintenance (Luria et al., 2016; Vogel \& Machizawa, 2004). The increasing CDA amplitude across set sizes was originally considered somewhat problematic for classic fixed resource models (Ma, Husain \& Bays, 2014) and has often been indicated as evidence in support of slot-like encoding (Vogel \& Machizawa, 2004). However, our model now provides a mechanism through which a resource-based model can predict increasing CDA amplitude: Having multiple items allows recruitment of composite-coding neurons. Recruitment of higher-order composites (eg. 3- or 4-item) may also occur. This may therefore increase downstream activity. 
The conjunctive code model

Furthermore, this model predicts differential effects of similarity and complexity on CDA amplitude. Specifically, the CDA amplitude should increase across set sizes for displays containing similar stimuli but remain relatively high for complex stimuli. This prediction is due to complex stimuli necessarily needing to draw on downstream visual areas, and therefore having a relatively high CDA even at low set sizes. However, for simple stimuli, these downstream areas will only be drawn upon when more items are encoded. Luria et al (2010) found overall higher CDA amplitudes for complex stimuli (polygons) which did not increase with set size but found increasing CDA amplitudes across set sizes for simple stimuli (colours). This is exactly what would be predicted from recruitment of higher-order composites. Additionally, Zhang et al (2016) also conducted a change detection task for oriented arrows, manipulating whether the orientations within a display were similar or dissimilar to one another. In addition to better performance for similar displays, they also found a positive slope between set size and CDA for the similar displays, but not for the dissimilar displays. These results can be re-interpreted under our theoretical framework: at larger set sizes, additional regions are drawn upon to represent the composite, leading to greater CDA amplitude. Because composites rely on the stimuli being in some sense similar, or groupable, the effect only becomes apparent for similar displays, but not dissimilar displays.

It may be possible that composite codes can be detectable in fMRI tasks. Lorenc et al (2020) may have recently identified regions that code for the composite of two orientations during WM maintenance. They trained a classifier to decode the fMRI signal from a task in which participants remembered the orientation of two sinusoidal gratings. They identified an area along the left intraparietal sulcus which appeared to uniquely code for both orientations. This may be the region where orientation composites are represented. Additionally, recent work has shown that the differences between colours during memory maintenance are represented in the parietal cortex, and can be decoded from fMRI data (Zhao et al., 2021). 
The conjunctive code model

One further consideration of our results is that set size effects are relatively easy to capture, and have been described by a large variety of competing models (examples include: Bays \& Husain, 2008; Cowan 2001; Lewandowsky \& Farrell, 2008b; Luck \& Vogel, 2013; Oberauer \& Lin, 2017; Oberauer et al., 2018; Pashler, 1988; van den Berg et al., 2012; van den Berg et al., 2015; Wilken \& Ma, 2004; Zhang \& Luck, 2008). Future work will be needed to test more diagnostic predictions for ruling out alternative models (although alternative explanations for increasing informational capacity are not necessarily mutually exclusive). For example, the resource rational (Sims, Jacobs \& Knill, 2012; van den Berg \& Ma, 2018) and neural coding models (Schneegans et al., 2020) predict increasing information capacity, but do not predict correlated errors or the similarity benefit. Additionally, different models which assume hierarchical information is encoded make competing predictions. For example, if summary statistics are encoded, we should expect to see biases towards the mean of the display (Brady \& Alvarez, 2011), but not the observed correlation in recall errors we have reported. However, again, it is possible that multiple types of hierarchical information can be extracted.

We believe that our results also have relevance to other existing debates in the WM literature. First, there is debate surrounding whether the precision of representations in WM is variable across items within- and between- displays (van den Berg et al., 2012). Variable precision models often assume different quantities of total resource across trials, perhaps due to fluctuations in attention. However, our model predicts variable precision while assuming a unitarily fixed resource capacity. Specifically, the precision with which an individual item in a study display is recalled will depend on its similarity to the other items in the display.

To summarise, we consider the well replicated finding from delayed reproduction tasks in which WM capacity, appears to increase across set sizes - a finding at odds with the classic view of a unitarily fixed WM capacity. We propose that this may be explained by the already well-understood hierarchical organisation of the visual system, in which downstream visual areas represent the 
The conjunctive code model

composites of lower level features. There is a growing body of evidence that visual WM maintenance makes use of the existing organisation of the visual system (e.g., Brady \& Störmer, 2021; Hedayati et al., 2021). One strength of this model is that it is based in biologically plausible mechanisms and our existing understanding of the visual system. We believe that understanding of visual WM will benefit greatly from a deeper understanding of how it relies on the existing organisation of the visual system. 
The conjunctive code model

\section{References}

Akaike, H. (1974). A new look at the statistical model identification. IEEE Transactions on Automatic Control, 19(6), 716-723.

Atkinson, R. C., \& Shiffrin, R. M. (1968). Human memory: A proposed system and its control processes. Psychology of Learning and Motivation, 2, 89-195.

Averbeck, B. B., Latham, P. E., \& Pouget, A. (2006). Neural correlations, population coding and computation. Nature Reviews Neuroscience, 7(5), 358-366.

Baddeley, A. (2000). The episodic buffer: a new component of working memory? Trends in Cognitive Sciences, 4(11), 417-423.

Baddeley, A. D., \& Hitch, G. (1974). Working memory. In Psychology of Learning and Motivation, 8, 47-89.

Bae, G. Y., \& Luck, S. J. (2017). Interactions between visual working memory representations. Attention, Perception, \& Psychophysics, 79(8), 2376-2395.

Bays, P. M. (2014). Noise in neural populations accounts for errors in working memory. Journal of Neuroscience, 34(10), 3632-3645.

Bays, P. M., \& Husain, M. (2008). Dynamic shifts of limited working memory resources in human vision. Science, 321(5890), 851-854.

Bays, P. M., Catalao, R. F., \& Husain, M. (2009). The precision of visual working memory is set by allocation of a shared resource. Journal of Vision, 9(10), 1-11.

Bergmann, J., Genç, E., Kohler, A., Singer, W., \& Pearson, J. (2016). Neural anatomy of primary visual cortex limits visual working memory. Cerebral Cortex, 26(1), 43-50.

Brady, T. F., \& Alvarez, G. A. (2011). Hierarchical encoding in visual working memory: Ensemble statistics bias memory for individual items. Psychological Science, 22(3), 384-392. 
The conjunctive code model

Brady, T. F., \& Alvarez, G. A. (2015). No evidence for a fixed object limit in working memory: Spatial ensemble representations inflate estimates of working memory capacity for complex objects. Journal of Experimental Psychology: Learning, Memory, and Cognition, 41(3), 921929.

Brady, T. F., Konkle, T., \& Alvarez, G. A. (2009). Compression in visual working memory: using statistical regularities to form more efficient memory representations. Journal of Experimental Psychology: General, 138(4), 487-502.

Brady, T. F., Störmer, V. S., \& Alvarez, G. A. (2016). Working memory is not fixed-capacity: More active storage capacity for real-world objects than for simple stimuli. Proceedings of the National Academy of Sciences, 113(27), 7459-7464.

Broadbent, D. E. (1958). Perception and Communication. Elsevier.

Chen, X., Li, B., \& Liu, Y. (2017). The impact of object complexity on visual working memory capacity. Psychology, 8(06), 929-937.

Christophel, T. B., Hebart, M. N., \& Haynes, J. D. (2012). Decoding the contents of visual short-term memory from human visual and parietal cortex. Journal of Neuroscience, 32(38), 1298312989.

Corbett, J. E. (2017). The whole warps the sum of its parts: Gestalt-defined-group mean size biases memory for individual objects. Psychological Science, 28(1), 12-22.

Cowan, N. (2001). The magical number 4 in short-term memory: A reconsideration of mental storage capacity. Behavioral and Brain Sciences, 24(1), 87-114.

Dai, M., Li, Y., Gan, S., \& Du, F. (2019). The reliability of estimating visual working memory capacity. Scientific Reports, 9(1), 1-8. 
The conjunctive code model

D'Esposito, M., \& Postle, B. R. (2015). The cognitive neuroscience of working memory. Annual Review of Psychology, 66, 115-142.

Emrich, S. M., Riggall, A. C., LaRocque, J. J., \& Postle, B. R. (2013). Distributed patterns of activity in sensory cortex reflect the precision of multiple items maintained in visual short-term memory. Journal of Neuroscience, 33(15), 6516-6523.

Endress, A. D., \& Potter, M. C. (2014). Large capacity temporary visual memory. Journal of Experimental Psychology: General, 143(2), 548-565.

Goodale, M. A., \& Milner, A. D. (1992). Separate visual pathways for perception and action. Trends in Neurosciences, 15(1), 20-25.

Gross, C. G., Rocha-Miranda, C. D., \& Bender, D. B. (1972). Visual properties of neurons in inferotemporal cortex of the macaque. Journal of Neurophysiology, 35(1), 96-111.

Güçlü, U., \& van Gerven, M. A. (2015). Deep neural networks reveal a gradient in the complexity of neural representations across the ventral stream. Journal of Neuroscience, 35(27), 1000510014.

Harrison, S. A., \& Tong, F. (2009). Decoding reveals the contents of visual working memory in early visual areas. Nature, 458(7238), 632-635.

Hedayati, S., O'Donnell, R., \& Wyble, B. (2021). The Memory for Latent Representations: An Account of Working Memory that Builds on Visual Knowledge for Efficient and Detailed Visual Representations. bioRxiv.

Hubel, D. H., \& Wiesel, T. N. (1959). Receptive fields of single neurones in the cat's striate cortex. The Journal of Physiology, 148(3), 574-591.

Jacobs, J. (1887). Experiments on" prehension". Mind, 12(45), 75-79. 
The conjunctive code model

Jiang, Y., Olson, I. R., \& Chun, M. M. (2000). Organization of visual short-term memory. Journal of Experimental Psychology: Learning, Memory, and Cognition, 26(3), 683-702.

Lee, S. H., Kravitz, D. J., \& Baker, C. I. (2013). Goal-dependent dissociation of visual and prefrontal cortices during working memory. Nature Neuroscience, 16(8), 997-999.

Lew, T. F., \& Vul, E. (2015). Ensemble clustering in visual working memory biases location memories and reduces the Weber noise of relative positions. Journal of Vision, 15(4), 1-14.

Lewandowsky, S., \& Farrell, S. (2008). Short-term memory: New data and a model. Psychology of Learning and Motivation, 49, 1-48.

Lin, P. H., \& Luck, S. J. (2009). The influence of similarity on visual working memory representations. Visual Cognition, 17(3), 356-372.

Lorenc, E. S., Vandenbroucke, A. R., Nee, D. E., de Lange, F. P., \& D’Esposito, M. (2020). Dissociable neural mechanisms underlie currently-relevant, future-relevant, and discarded working memory representations. Scientific Reports, 10(1), 1-17.

Luck, S. J., \& Vogel, E. K. (1997). The capacity of visual working memory for features and conjunctions. Nature, 390(6657), 279-281.

Luck, S. J., \& Vogel, E. K. (2013). Visual working memory capacity: from psychophysics and neurobiology to individual differences. Trends in Cognitive Sciences, 17(8), 391-400.

Luria, R., Balaban, H., Awh, E., \& Vogel, E. K. (2016). The contralateral delay activity as a neural measure of visual working memory. Neuroscience \& Biobehavioral Reviews, 62, 100-108.

Luria, R., Sessa, P., Gotler, A., Jolicœur, P., \& Dell'Acqua, R. (2010). Visual short-term memory capacity for simple and complex objects. Journal of Cognitive Neuroscience, 22(3), 496-512.

Ma, W. J., Husain, M., \& Bays, P. M. (2014). Changing concepts of working memory. Nature Neuroscience, 17(3), 347-356. 
The conjunctive code model

Magnussen, S., Greenlee, M. W., \& Thomas, J. P. (1996). Parallel processing in visual short-term memory. Journal of Experimental Psychology: Human Perception and Performance, 22(1), 202-212.

Manohar, S. G., Zokaei, N., Fallon, S. J., Vogels, T. P., \& Husain, M. (2019). Neural mechanisms of attending to items in working memory. Neuroscience \& Biobehavioral Reviews, 101, 1-12.

Miller, G. A. (1956). The magical number seven, plus or minus two: Some limits on our capacity for processing information. Psychological Review, 63(2), 81-97.

Moreno-Bote, R., Beck, J., Kanitscheider, I., Pitkow, X., Latham, P., \& Pouget, A. (2014). Informationlimiting correlations. Nature Neuroscience, 17(10), 1410-1417.

Oberauer, K. (2002). Access to information in working memory: exploring the focus of attention. Journal of Experimental Psychology: Learning, Memory, and Cognition, 28(3), 411-421.

Oberauer, K., \& Lin, H. Y. (2017). An interference model of visual working memory. Psychological Review, 124(1), 21-59.

Oberauer, K., Lewandowsky, S., Awh, E., Brown, G. D., Conway, A., Cowan, N., ... \& Ward, G. (2018). Benchmarks for models of short-term and working memory. Psychological Bulletin, 144(9), 885-958.

Orhan, A. E., \& Jacobs, R. A. (2013). A probabilistic clustering theory of the organization of visual short-term memory. Psychological Review, 120(2), 297-328.

Pashler, H. (1988). Familiarity and visual change detection. Perception \& Psychophysics, 44(4), 369378.

Pasternak, T., \& Greenlee, M. W. (2005). Working memory in primate sensory systems. Nature Reviews Neuroscience, 6(2), 97-107. 
The conjunctive code model

Pessoa, L., Gutierrez, E., Bandettini, P. A., \& Ungerleider, L. G. (2002). Neural correlates of visual working memory: fMRI amplitude predicts task performance. Neuron, 35(5), 975-987.

Peters, B., Rahm, B., Kaiser, J., \& Bledowski, C. (2019). Differential trajectories of memory quality and guessing across sequential reports from working memory. Journal of Vision, 19(7), 1-14.

Peterson, L., \& Peterson, M. J. (1959). Short-term retention of individual verbal items. Journal of Experimental Psychology, 58(3), 193-198.

Phillips, J. J., Shiffrin, R. M., Atkinson, R. C. (1965). Effects of list length on short-term memory. Journal of Verbal Learning and Verbal Behavior, 6(3), 303-311.

Pylyshyn, Z. (1989). The role of location indexes in spatial perception: A sketch of the FINST spatialindex model. Cognition, 32(1), 65-97.

Riggall, A. C., \& Postle, B. R. (2012). The relationship between working memory storage and elevated activity as measured with functional magnetic resonance imaging. Journal of Neuroscience, 32(38), 12990-12998.

Schneegans, S., \& Bays, P. M. (2017). Neural architecture for feature binding in visual working memory. Journal of Neuroscience, 37(14), 3913-3925.

Schneegans, S., Taylor, R., \& Bays, P. M. (2020). Stochastic sampling provides a unifying account of visual working memory limits. Proceedings of the National Academy of Sciences, 117(34), 20959-20968.

Schurgin, M. W., \& Brady, T. F. (2019). When "capacity" changes with set size: Ensemble representations support the detection of across-category changes in visual working memory. Journal of Vvision, 19(5), 1-13.

Serences, J. T., Ester, E. F., Vogel, E. K., \& Awh, E. (2009). Stimulus-specific delay activity in human primary visual cortex. Psychological Science, 20(2), 207-214. 
The conjunctive code model

Seung, H. S., \& Sompolinsky, H. (1993). Simple models for reading neuronal population codes. Proceedings of the National Academy of Sciences, 90(22), 10749-10753.

Shannon, C. E. (1948). A mathematical theory of communication. The Bell system technical journal, 27(3), 379-423.

Sims, C. R., Jacobs, R. A., \& Knill, D. C. (2012). An ideal observer analysis of visual working memory. Psychological Review, 119(4), 807-830.

Son, G., Oh, B. I., Kang, M. S., \& Chong, S. C. (2020). Similarity-based clusters are representational units of visual working memory. Journal of Experimental Psychology: Learning, Memory, and Cognition, 46(1), 46-59.

Sperling, G. (1960). The information available in brief visual presentations. Psychological Monographs: General and Applied, 74(11), 1-29.

Super, H., Spekreijse, H., \& Lamme, V. A. (2001). A neural correlate of working memory in the monkey primary visual cortex. Science, 293(5527), 120-124.

Swan, G., \& Wyble, B. (2014). The binding pool: A model of shared neural resources for distinct items in visual working memory. Attention, Perception, \& Psychophysics, 76(7), 2136-2157.

Treisman, A. M., \& Gelade, G. (1980). A feature-integration theory of attention. Cognitive Psychology, 12(1), 97-136.

Treisman, A. (1988). Features and objects: The fourteenth Bartlett memorial lecture. The Quarterly Journal of Experimental Psychology Section A, 40(2), 201-237.

Van den Berg, R., \& Ma, W. J. (2018). A resource-rational theory of set size effects in human visual working memory. ELife, 7, e34963. 
The conjunctive code model

Van den Berg, R., Shin, H., Chou, W. C., George, R., \& Ma, W. J. (2012). Variability in encoding precision accounts for visual short-term memory limitations. Proceedings of the National Academy of Sciences, 109(22), 8780-8785.

Van den Berg, R., Awh, E., \& Ma, W. J. (2014). Factorial comparison of working memory models. Psychological Review, 121(1), 124-149.

Vogel, E. K., \& Machizawa, M. G. (2004). Neural activity predicts individual differences in visual working memory capacity. Nature, 428(6984), 748-751.

Wandell, B. A., Dumoulin, S. O., \& Brewer, A. A. (2007). Visual field maps in human cortex. Neuron, 56(2), 366-383.

Wilken, P., \& Ma, W. J. (2004). A detection theory account of change detection. Journal of Vision, 4(12), 1120-1135.

Woodman, G. F., Vecera, S. P., \& Luck, S. J. (2003). Perceptual organization influences visual working memory. Psychonomic Bulletin \& Review, 10(1), 80-87.

Xu, Y., \& Chun, M. M. (2006). Dissociable neural mechanisms supporting visual short-term memory for objects. Nature, $440(7080), 91-95$.

Zhang, W., \& Luck, S. J. (2008). Discrete fixed-resolution representations in visual working memory. Nature, 453(7192), 233-235.

Zhang, Q., Li, S., Wang, X., \& Che, X. (2016). The effects of direction similarity in visual working memory: Behavioural and event-related potential studies. Quarterly Journal of Experimental Psychology, 69(9), 1812-1830.

Zhao, Y., Chanales, A. J., \& Kuhl, B. A. (2021). Adaptive memory distortions are predicted by feature representations in parietal cortex. Journal of Neuroscience. In press. 